\title{
Prática de atividade física habitual entre adolescentes com deficiência visual
}

CDD. 20.ed. 613.7

796.019

\author{
Bruna Barboza SERON* \\ Giovanna Carla INTERDONATO* \\ Clovis Corrêa LUIZ J UNIOR* \\ Márcia GREGUOL*
}

*Centro de Educação

Física e Esporte, Uni-

versidade Estadual de

Londrina.

\begin{abstract}
Resumo
A prática de atividade física é reconhecida como uma potente ferramenta na prevenção de doenças crônico-degenerativas, entretanto ainda se observa um elevado índice de sedentarismo na população, especialmente na parcela composta por pessoas com deficiência. 0 objetivo desse estudo foi analisar a prática habitual de atividade física em adolescentes com deficiência visual. Para tanto, 16 adolescentes com deficiência visual (idade $=12,81 \pm 2,07$ anos) responderam a questionários e utilizaram pedômetro por quatro dias. Foi utilizada estatística descritiva, teste $t$ independente e correlação, adotando-se em todas as situações significância de alfa $<0,05$. Os dados mostraram que o nível de atividade física entre os adolescentes foi abaixo do recomendável, sendo que as meninas e aqueles com deficiência total apresentaram piores resultados. Os adolescentes mostraram-se significativamente mais ativos durante a semana. Desta forma, conclui-se que os adolescentes pesquisados eram insuficientemente ativos, necessitando de mais programas de intervenção.
\end{abstract}

UNITERMOS: Pedômetro; Deficiência visual; Sedentarismo.

\section{Introdução}

Embora sejam amplamente divulgados na literatura os benefícios da prática da atividade física como forma de prevenir ou reduzir os fatores de risco ligados a doenças e agravos não transmissíveis, observa-se ainda elevado índice de sedentarismo entre indivíduos dos mais diversos grupos etários (Alhassan \& Robinson, 2010; Peixoto, Benício \& JARDIM, 2007). Especificamente na idade adulta, alguns autores associam tal índice ao comportamento sedentário observado na infância e adolescência (Guedes \& Guedes, 2006). Acompanhamentos longitudinais sugerem que adolescentes menos ativos fisicamente apresentam maior predisposição a tornarem-se adultos sedentários (BAUMAN, 2004). No entanto, ainda é possível notar uma carência de estudos relacionados à prática habitual de atividade física envolvendo crianças e adolescentes com alguma deficiência.

Indivíduos com deficiência são percebidos pela sociedade como dependentes de cuidados e proteção e historicamente têm sido excluídos das oportunidades de acesso aos programas oferecidos para pessoas sem deficiência (Lieberman, 2005; Noce, Simim \& Mello, 2009). Como consequência, apesar dos avanços legais das últimas décadas, ainda se percebe grande tendência desta população ao isolamento social e à não participação em atividades promovidas com o objetivo de aprimoramento da saúde e qualidade de vida, tais como programas de atividades físicas. Comparados com os demais indivíduos, aqueles com deficiência demonstram com maior frequência comportamentos sedentários, prevalência de sobrepeso e obesidade e níveis mais precários de aptidão física (Cervantes \& Porreta, 2010).

Kodish, Kulinna, Martin, Pangrazi e Darst (2006) destacam a falta de oportunidades de jovens com deficiência de participarem em programas organizados de atividade física, inclusive durante as práticas da educação física escolar. Tal fato se deve sobretudo à falta de capacitação dos professores e de 
recursos materiais e espaços apropriados. Ainda como fator agravante, segundo os autores, as oportunidades de vivências de atividade física fora dos programas formais também são reduzidas. Esta dificuldade se deveria especialmente à falta de acessibilidade arquitetônica e ao desconhecimento dos pais sobre as possibilidades de seus filhos e os possíveis benefícios que estes obteriam com tal prática. Esta situação certamente acarreta grande risco que estes jovens tornem-se adultos sedentários e, por consequência, mais propensos ao desenvolvimento de condições de risco, tais como problemas ósseos e musculares, obesidade e maior tendência de desenvolvimento de doenças cardiovasculares e perda de autonomia para a realização das atividades do dia-a-dia. Tal perda de autonomia pode ser ainda mais evidenciada quando se analisam as atividades desempenhadas por indivíduos com deficiência visual, uma vez que grande parte de nossa comunicação com o ambiente se dá pelo sentido da visão.

BitTenCourt e HoEHne (2006) afirmam que a dificuldade de desempenhar as tarefas pessoais e a dependência são especialmente devastadores para o indivíduo que possui deficiência visual, pois a visão é considerada a grande promotora da integração do indivíduo em atividades motoras, perceptivas e mentais e a perda da mesma pode provocar marcantes alterações, diminuindo sua capacidade de adaptação na sociedade.

Em um estudo realizado com crianças e adolescentes com diversos tipos de deficiências, com idades entre seis e 20 anos, LONGMUIR e BAR-OR (2000) verificaram que aqueles que possuíam deficiência visual apresentavam níveis mais baixos de atividade física habitual quando comparados a outros com deficiências motoras. Além disso, aqueles com deficiência visual percebiam maior limitação para a prática de atividades físicas junto a colegas sem deficiências, o que talvez pudesse aumentar sua falta de motivação para realizar tais atividades, dificultando assim sua participação ativa.

Estudos mostram que crianças e adolescentes com deficiência visual apresentam níveis de aptidão física relacionada à saúde abaixo da média de jovens videntes da mesma faixa etária (Greguol \& De Rose Junior, 2009; Korach, Tennenbaum, Schnitzer \& Ornoy, 2000; Lopes, Kitadai \& Okai, 2004). Entretanto, existe também certa unanimidade entre os autores de que uma estimulação adequada e precoce, além da possibilidade de experimentação ampla e variada do ambiente, pode resolver ou pelo menos reduzir em grande parte o atraso existente.

Observa-se na literatura que existem poucas informações acerca dos comportamentos relacionados à saúde de jovens com deficiência, em especial envolvendo indicadores sobre os níveis de atividade física habitual. Assim, o objetivo deste estudo foi quantificar a prática de atividade física entre adolescentes com deficiência visual do município de Londrina - PR, destacando sua distribuição ao longo da semana. Ainda, objetivou-se identificar possíveis variáveis correlacionadas à prática de atividade física pelos adolescentes.

\section{Materiais e métodos}

\section{Amostra}

A amostra foi composta por 16 adolescentes do gênero masculino e feminino, com idade média de 12,81 anos $( \pm 2,07)$, todos com deficiência visual. Para serem inclusos no estudo, os adolescentes deveriam apresentar diagnóstico de deficiência visual congênita, sendo esta classificada como cegueira ou visão subnormal, ser frequentadores do Instituto Londrinense de Instrução e Trabalho para Cegos (ILITC) e estar matriculados em escolas regulares da cidade de Londrina/PR. Como critérios de exclusão, não participaram do estudo adolescentes que estivessem fora do sistema regular de ensino ou que apresentassem deficiências motoras ou intelectuais associadas. Todos os sujeitos, assim como seus responsáveis legais, depois de esclarecidos os procedimentos de pesquisa, assinaram um Termo de Consentimento Livre e Esclarecido. Este estudo obteve aprovação do Comitê de Ética em Pesquisa com Seres Humanos da Universidade Estadual de Londrina (Parecer 15640/2007).

\section{Procedimentos}

Os adolescentes responderam um questionário inicial, com informações sobre o gênero, idade e classificação da deficiência em cegueira ou visão subnormal. Em seguida, foram distribuídos questionários aos adolescentes e aos seus pais, nos quais deveriam dizer o quanto acreditavam que a prática de atividade física era importante para a saúde positiva (nada importante, pouco importante ou muito importante). No questionário dos pais existia uma questão adicional abordando o quanto incentivavam seus filhos a 
praticarem atividade física (nada, pouco ou muito).

Para a classificação econômica das famílias dos entrevistados, recorreu-se às diretrizes propostas pela Associação Nacional de Empresas de Pesquisa (ANEP, 2000). Mediante pontuações computadas em cada um dos itens considerados, foi definida a classe econômica familiar categorizada do menor nível (classe E) para o maior nível (classe A). As classes A1 e A2 foram agrupadas genericamente como A, assim como as classes B1 e B2 em B.

Para a análise dos níveis de atividade física foi utilizado o International Physical Activity Questionnaire/IPAQ - versão curta (BASSETT, 2003), com perguntas relacionadas ao tempo gasto com a atividade no intervalo de sete dias passados desde a data de aplicação do instrumento. A aplicação dos questionários foi feita por meio de leitura em voz alta para os entrevistados e os mesmos foram respondidos pelos adolescentes.

Ainda com o objetivo de mensurar a atividade física habitual dos participantes do estudo, foram utilizados pedômetros da marca Digiwalker ( $S W$ 200), a fim de analisar o número de passos dados ao longo do tempo, através do registro das oscilações verticais do corpo. O número de passos reflete, sobretudo, um aspecto relevante da quantidade da atividade física. Assim, cada sujeito foi monitorado durante quatro dias, dois durante a semana, sábado e domingo. Os adolescentes, assim como seus responsáveis, receberam informações sobre o manuseio adequado do pedômetro, que foi colocado na cintura e retirado apenas para dormir, tomar banho e trocar de roupa. Foi entregue uma ficha de registro para que fosse anotado o número do passos ao final de cada dia.

\section{Análise estatística}

O tratamento estatístico das informações foi realizado por meio do programa Statistical Package for the Social Science (SPSS), versão 15.0. Após verificada a normalidade dos dados por meio do teste de Shapiro-Wilk, foi utilizada a estatística descritiva para caracterização da amostra. Utilizou-se teste $t$ independente para comparação dos dados da pedometria entre adolescentes com deficiência visual total e parcial e entre gêneros e teste $t$ dependente para comparar os resultados da pedometria durante os dias da semana e do final de semana, assim como entre os resultados do sábado e domingo. Para verificar possíveis correlações entre os resultados da pedometria com o nível socioeconômico e idade, realizou-se teste de correlação de Pearson. Como o número de $\mathrm{MET}$ s do IPAQ foi a única variável que não mostrou distribuição normal, utilizou-se o teste de correlação de Spearman para analisar sua possível relação com os dados da pedometria. Em todas as situações o nível de significância adotado foi de alfa $<0,05$.

\section{Resultados}

Foram no total avaliados 16 adolescentes com deficiência visual, sendo nove do gênero feminino, com idade média de $13(+1,87)$ anos, e sete do gênero masculino, com idade média de $12,57(+2,44)$ anos.

A TABELA 1 traz as informações da amostra quanto à classificação da deficiência e nível socioeconômico.

De acordo com os dados do IPAQ, um adolescente foi classificado como sedentário, 12 como irregularmente ativos e três como ativos. Os resultados descritivos referentes aos dados da pedometria durante os dias da semana, ao final de semana, assim como os valores de METs verificados pelo IPAQ encontram-se na TABELA 2.
TABELA 1 - Dados descritivos da amostra relacionados à classificação da deficiência e nível socioeconômico.

\begin{tabular}{lcc}
\hline Variável & Cegueira & $\begin{array}{c}\text { Número } \\
\text { de sujeitos }\end{array}$ \\
\hline Classificação & 4 \\
Nível socioeconômico & A & 12 \\
& B & 1 \\
& C & 4 \\
& D & 9 \\
\hline
\end{tabular}


TABELA 2 - Valores descritivos das medidas de atividade física habitual.

\begin{tabular}{lcc}
\hline \multicolumn{1}{c}{ Variável } & Média & Desvio Padrão \\
\hline Número de passos durante a semana & 7389,8 & 684,9 \\
Número de passos ao final de semana & 6038,2 & 843 \\
Número de passos apenas ao sábado & 7524 & 807 \\
Número de passos apenas ao domingo & 5565,9 & 928,1 \\
MET (IPAQ) & 2822 & 2324,4 \\
\hline
\end{tabular}

Quando se comparou os dados obtidos pelo pedômetro entre a média dos dias da semana e a média do final de semana, encontrou-se diferença significativa $(p=0,031)$, indicando que durante a semana os adolescentes caminharam mais que ao final de semana. Além disso, ao comparar a média de passos do sábado e domingo, foi encontrada diferença significativa $(p=0,015)$, sendo que no domingo os adolescentes caminharam significativamente menos que no sábado.

Quanto aos dados obtidos na comparação entre os adolescentes com deficiência visual total e parcial nos resultados da pedometria, não se encontrou diferença significativa ( $p=0,058)$. Ao considerar comparaçôes entre gêneros na pedometria, houve diferença significativa, sendo que as meninas caminharam significativamente menos do que os meninos $(\mathrm{p}=0,033)$.

Foram levantadas ainda correlaçóes entre os dados da pedometria, o número de METs demonstrado pelo IPAQ, a idade e NSE. Os resultados evidenciaram uma correlação significativa $(r=0,514 ; \mathrm{p}=0,042)$ entre o número total de passos e o NSE, demonstrando que quanto mais elevada a classe socioeconômica, menos o adolescente caminhava. Houve ainda uma correlação significativa entre os dados apontados pelos adolescentes no IPAQ em número de METs e os resultados da pedometria $(r=0,736 ; \mathrm{p}=0,02)$. Entretanto, pelos resultados do IPAQ, apenas um dos pesquisados foi classificado como sedentário. No entanto, ao analisar os resultados da pedometria, a maioria dos adolescentes apresentou valores abaixo dos indicados pela literatura para uma saúde positiva (Lieberman, Stuart, Hand \& Robinson, 2006). A correlação dos dados da pedometria com a idade dos sujeitos mostrou-se negativa, porém sem significância estatística $(r=-0,476 ; p=0,07)$.

Quanto à importância e incentivo para a prática de atividade física, $100 \%$ dos pais entrevistados afirmaram acreditar que esta era importante para a saúde de seus filhos, porém apenas 10 deles $(62,7 \%)$ incentivavam de maneira constante tal prática, enquanto seis $(37,5)$ incentivavam pouco. Entre os adolescentes, 13 $(81,3 \%)$ disseram acreditar que a prática de atividade física é muito importante para a saúde, dois $(12,5 \%)$ que é um pouco importante e um deles $(6,3 \%)$ afirmou que não achava nada importante.

\section{Discussão}

Ao analisar os resultados obtidos no presente estudo, verifica-se que a média de passos dos adolescentes pesquisados ficou muito aquém dos padróes ideais recomendados pelo PrESIDENT's COUNCIL ON PHYSICAL FITNESS AND SPORTS (2004) que estabelece um mínimo de 11.000 passos para meninas e 13.000 para meninos nesta faixa etária. Da mesma maneira, LIEBERMAN et al. (2006) ao avaliarem 22 crianças com deficiência visual (nove a 13 anos) com pedometria durante sete dias consecutivos, obtiveram valores inferiores aos recomendados, com médias de 9686 passos por dia para as meninas e 9770 para os meninos.

Embora a pedometria avalie apenas as informações obtidas durante a caminhada, ela é um indicativo relevante que reflete a prática de atividade física habitual dos indivíduos. Assim, é possível afirmar que os adolescentes pesquisados encontram-se abaixo dos níveis de atividade física considerados compatíveis com a manutenção da saúde positiva. Tais níveis baixos podem ser creditados às poucas oportunidades de vivenciar momentos de lazer ativo e à insegurança nos deslocamentos e passividade motora muitas vezes observada por indivíduos com deficiência visual. Além disso, a superproteção por parte da família, aliada às poucas oportunidades de engajamento em programas de atividade física regular, pode ser um fator explicativo para os níveis insuficientes verificados. 
Apesar da elevada correlação observada entre os dados da pedometria e o número de METs obtido pelo IPAQ, foi possível verificar divergência entre os resultados dos dois instrumentos, uma vez que, pelo questionário, apenas um dos adolescentes seria classificado como sedentário. Já pela pedometria, nenhum dos adolescentes pesquisados atingiu os parâmetros mínimos recomendáveis. Essa divergência pode ser devida ao fato de que os adolescentes com deficiência visual apresentaram dificuldade em recordar as atividades realizadas ao longo da semana, além de pouca familiarização em responder este tipo de instrumento.

Em um estudo buscando correlacionar os resultados do IPAQ com os da pedometria, Benedetti, Mazo e BARROS (2004) também evidenciaram baixa concordância entre os resultados. Segundo os autores, o questionário deveria ser utilizado apenas em situações nas quais a discriminação de níveis gerais de atividade física fosse suficiente ou então quando o número da amostra estudada fosse muito grande. Além disso, a aplicação de questionários recordatórios com adolescentes pode ser mais passível de erros pela dificuldade destes em relatar as atividades realizadas previamente. Assim, não seria recomendável a utilização do instrumento em estudos com pequeno número de pessoas, nos quais é possível fazer a monitoração direta das atividades, o que daria mais confiabilidade aos resultados.

Na comparação dos resultados da pedometria durante a semana e ao final de semana, observou-se que os adolescentes mostraram níveis significativamente mais elevados de atividade física durante a semana. Este resultado provavelmente se deve ao fato de que, durante a semana, os adolescentes possuem as atividades da escola e do ILITC, que demandam maiores deslocamentos e, consequentemente, geram impacto positivo na quantidade de atividade física total realizada. Já ao final de semana, a atividade física seria principalmente creditada às atividades de lazer, que, para pessoas com deficiência, em geral tendem a ser mais passivas.

Dentro do final de semana, o nível de atividade física mensurado ao domingo foi significativamente inferior ao do sábado. Lopes, Maia, Oliveira, Seabra e GARGANTA (2003) analisando o nível habitual de atividade física de adolescentes sem deficiência de ambos os gêneros durante uma semana, também observou resultados inferiores ao final de semana, especialmente no domingo quando comparado ao sábado. Como mencionado anteriormente, jovens com deficiência visual tendem a ser excessivamente protegidos pelos pais e raramente são estimulados a momentos de lazer ativo, o que pode explicar o maior nível de inatividade verificado aos finais de semana, especialmente no domingo. Em geral, também pela falta de programas direcionados e espaços acessíveis, estes jovens no seu tempo livre manifestam comportamentos mais passivos, optando por atividades envolvendo computador, videogame ou televisão.

Ao comparar os resultados obtidos pelos adolescentes com deficiência visual total e parcial, não foram evidenciadas diferenças significativas nos resultados da pedometria $(0,058)$. Talvez o número reduzido de adolescentes com deficiência total ( $\mathrm{n}=$ 4) tenha prejudicado as análises, uma vez que era esperado, pela maior insegurança em se deslocar, que os níveis de atividade física dos indivíduos com perda total da visão fossem menores do que daqueles com visão residual. SKAGGS e HOOPER (1996), ao analisarem variáveis da aptidão física relacionada à saúde de adolescentes com deficiência visual, verificaram que, quanto mais grave a perda da visão, mais precários eram os resultados. Assim os autores evidenciaram que os sujeitos com deficiência total possuíam índices mais baixos de aptidão física quando comparados com aqueles com deficiência parcial.

Já na comparação entre os gêneros, neste estudo foi observado que os meninos apresentaram níveis de atividade física significativamente superiores aos das meninas. Outros estudos na literatura têm destacado que, entre adolescentes, os meninos são mais fisicamente ativos do que as meninas (GUEDES \& Guedes,2006; Hallal, 2005; Lopes et al., 2003).

De acordo com Guedes e Guedes (2006), desde muito jovens, a participação dos rapazes em atividades esportivas e em esforços físicos mais intensos é valorizada mais positivamente que a das moças. Lima, Fermino, Seabra, Garganta e Maia (2010), em uma revisão sistemática da literatura abordando padrôes de atividade física de jovens, ressaltam que os meninos são em geral mais fisicamente ativos do que as meninas, além de despenderem mais tempo de seu dia em atividades vigorosas.

Embora todos os adolescentes pesquisados na amostra frequentassem a mesma instituição e fossem submetidos a atividades semelhantes, os melhores resultados verificados entre os meninos podem evidenciar que estes são mais estimulados pelos pais a comportamentos mais ativos e, portanto, menos superprotegidos do que as meninas.

Não foi observada correlação significativa entre o número de passos registrado pela pedometria e a idade dos adolescentes, embora esta tenha exibido uma tendência negativa, ou seja, quanto mais velho o adolescente menor o nível de atividade 
física registrado. Provavelmente o número reduzido de adolescentes em cada grupo etário tenha sido um fator que prejudicou as análises. Ainda assim, esta tendência pode ser explicada pelo maior comprometimento dos adolescentes mais velhos com as atividades escolares, assim como a preferência deste grupo por comportamentos mais passivos em seus momentos de lazer.

$\mathrm{Na}$ correlação entre os dados da pedometria e o nível socioeconômico, foi observada relação significativa, sendo que, quanto mais elevado o NSE, menor o número de passos registrado pelo adolescente, ou seja, menor o nível de atividade física. Este fato pode encontrar explicação, sobretudo, na forma de deslocamento dos adolescentes de suas casas para a escola e para o ILITC. Os adolescentes de NSE mais baixo em geral deslocam-se caminhando de suas casas para a escola, enquanto aqueles de níveis mais elevados fazem o deslocamento utilizando automóvel. Como os adolescentes com deficiência visual raramente participam de programas de atividade física fora de sua escola ou instituto, o tempo de caminhada gasto em seu deslocamento gera impacto significativo na quantidade total de atividade física realizada durante o dia.

Matsudo, Matsudo, Araújo, Andrade, AnDrade, Oliveira e Braggion (2002), analisando o nível de atividade física habitual de adolescentes da cidade de São Paulo - SP por meio de questionário recordatório (IPAQ), verificaram menores níveis entre os adolescentes das classes socioeconômicas extremas, ou seja, A e E. A classe A foi a que registrou maior prevalência de sedentarismo de acordo com as respostas obtidas.

Já Nunes, Figueiroa e Alves (2007), ao investigarem o estilo de vida e a prevalência de sobrepeso e obesidade de adolescentes com diferentes condiçóes socioeconômicas da cidade de Campina Grande - PB, obtiveram resultados diferentes. Embora tenham verificado maior prevalência de sobrepeso e obesidade entre os adolescentes das classes A1, A2 e B1, também neste grupo observaram maiores níveis de prática de atividade física no lazer. Uma justificativa para esta diferença pode ser atribuída ao instrumento utilizado para avaliar a atividade física dos adolescentes, uma vez que foram apenas investigadas as atividades realizadas no lazer, sem prever aquelas realizadas em outros momentos.

Assim, para os adolescentes que se deslocam no dia-a-dia utilizando automóvel, a falta de oferta de programas de atividade física direcionados exerce um impacto ainda mais negativo nos níveis observados de atividade física. Com a opção frequente por momentos de lazer predominantemente passivos, estes jovens assumem um estilo de vida cada vez mais sedentário, o que gera risco aumentado de sedentarismo na idade adulta.

Com relação à percepção dos pais sobre a importância da atividade física para a saúde dos filhos, observa-se que a maioria a julga importante, o que certamente reflete na crença dos adolescentes, embora os indicadores de prática não tenham sido considerados dentro dos padrôes recomendáveis. StuART, Lieberman e Hand (2006) analisaram 25 jovens com deficiência visual e seus pais e verificaram que as crenças sobre a importância da prática de atividade física dos filhos seguiam a mesma orientação daquela exibida pelos pais.

WENTHE, JANZ e LEVY (2009) analisaram o nível de atividade física habitual de 205 adolescentes e observaram que o apoio dos pais foi considerado um determinante fundamental para a prática de atividade física, demonstrando que o suporte familiar é de grande relevância para a adesão e aderência de adolescentes a comportamentos considerados compatíveis com a saúde positiva. No presente estudo, embora todos os pais dos adolescentes participantes acreditassem que a prática de atividade física era benéfica para a saúde, apenas $62,7 \%$ afirmou incentivar de maneira frequente este hábito em seus filhos. Apesar disto, fica evidenciado que já existe um conhecimento por parte dos adolescentes e seus pais sobre a importância de se praticar atividade física, embora tal conhecimento ainda deva ser convertido em ação.

O número reduzido da amostra no presente estudo, especialmente de adolescentes com deficiência total, certamente é um fator que dificulta a avaliação dos dados obtidos nas análises. Entretanto, ainda que poucos adolescentes tenham sido pesquisados, o número da amostra é representativo da população de jovens com deficiência visual da cidade de Londrina (em torno de 150 adolescentes com algum tipo de deficiência inseridos na rede regular de ensino do município, segundo dados do site Dia a Dia Educação, da Secretaria Municipal de Educação).

Mesmo com os avanços recentes da legislação no que tange a garantia de acesso de jovens com deficiência aos programas de atividade esportiva e de educação física na escola regular, é possível observar que na prática esta situação ainda parece distante de se concretizar. Por diversas razôes, que variam desde a falta de capacitação docente até as condiçōes precárias de acessibilidade, jovens com deficiência ainda têm 
dificuldade em inserir-se de maneira irrestrita em tais programas, exibindo, dessa forma, elevada tendência a um estilo de vida sedentário. Este fato alarmante sugere que mais estudos ainda são necessários abordando esta população específica, para que ações intervencionistas possam ser elaboradas com base em parâmetros confiáveis, de modo a contribuir para o desenvolvimento adequado dos jovens com deficiência e a formação de adultos com comportamentos comprometidos com a manutenção da boa saúde e qualidade de vida.

\title{
Conclusão
}

Pelos dados obtidos pode-se concluir que a quantidade de atividade física realizada pelos adolescentes com deficiência visual encontra-se abaixo dos parâmetros estabelecidos como recomendáveis para a saúde, sendo que a situação é ainda mais grave aos finais de semana. Na comparação entre os gêneros, conclui-se que as meninas são significativamente menos ativas que os meninos. Quanto à importância creditada à prática de atividade física, apesar de poucos pais serem fisicamente ativos, todos afirmaram que tal prática é de grande relevância para a saúde e que, portanto, incentivam seus filhos a realizá-la.

\begin{abstract}
Habitual physical activity among adolescents with visual impairments

Physical activity practice is known as a great tool in chronic diseases prevention, however we can observe a high prevalence of sedentary habits, especially among people with disability. The purpose of this study was to analyze habitual physical activity practice in adolescents with visual impairment. For that, 16 adolescents with visual impairment (age $=12.81 \pm 2.07$ years old) answered a questionnaire and used pedometer for four days. It was used descriptive statistic, independent $T$ test and correlation, in every case with significance level alpha $<0.05$. Results showed that physical activity level among adolescents was often below the recommended parameters. Girls and those with total visual impairment presented worse results. Adolescents exhibited higher physical activity level in week days. Thus, we can conclude that adolescents were insufficiently actives, what indicates the necessity of more interventions.
\end{abstract}

UnITERMS: Pedometer; Visual impairment; Sedentary.

\section{Resumen}

La actividad física habitual entre los adolescentes con discapacidad visual

La actividad física es reconocida como una herramienta en la prevención de las enfermedades crónicas degenerativas, sin embargo, ha observado una alta tasa de inactividad de la población, sobre todo en la parte compuesta por personas con discapacidad. El objetivo de este estudio fue analizar la práctica de actividad física en adolescentes con discapacidad visual. Para ello, 16 adolescentes con discapacidad visual (edad $=12,81 \pm 2,07$ años) completaron cuestionarios y se utilizó podómetro durante 4 días. Fue utilizada estadistica descriptiva, prueba t independiente y correlación, importancia de la alfa $<0,05$. Los datos mostraron que el nivel de actividad física entre los adolescentes fue menor de lo recomendado, y las niñas y las personas con discapacidad tienen los peores resultados globales. Los adolescentes fueron significativamente más activos durante la semana. Por lo tanto, se concluye que los adolescentes encuestados no eran suficientemente activos, que exige más programas de intervención.

Palabras clave: Podómetro; Discapacidad visual; Actividad física. 


\section{Referências}

ALHASSAN S.; ROBINSON T. N. Defining accelerometer thresholds for physical activity in girls using ROC analysis. Journal of Physical Activity and Health, Champaign, v.7, p.45-53, 2010. ASSOCIAÇÃO NACIONAL DE EMPRESAS DE PESQUISA (ANEP). Critério de classificação econômica no Brasil. São Paulo: Associação Nacional de Empresas de Pesquisa, 2000.

BASSETT, D.R. International physical activity questionnaire: 12 -country reliability and validity. Medicine $\&$ Science in Sports \& Exercise, Madison, v.35, n.8, p.1396, 2003.

BAUMAN, A.E. Updating the evidence that physical activity is good for health: an epidemiological review. 2000-2003. Journal of Sports Science and Medicine, Bursa, v.7, n.1, p.6-19, 2004.

BENEDETTI, T.B.; MAZO, G.Z.; BARROS, M.V.G. Aplicação do questionário internacional de atividades físicas para avaliação do nível de atividades físicas de mulheres idosas: validade concorrente e reprodutibilidade teste-reteste. Revista Brasileira de Ciência do Movimento, São Caetano do Sul, v.12, n.1, p.25-34, 2004.

BITTENCOURT, Z.L.; HOEHNE, E.L. Qualidade de vida de deficientes visuais. Medicina, Ribeirão Preto, v.39, n.2, p.260-4, 2006.

CERVANTES, C.M.; PORRETA, D.L. Physical activity measurement among individuals with disabilities: a literature review. Adapted Physical Activity Quarterly, Champaign, v.27, p.173-90, 2010.

GREGUOL, M.; De ROSE JUNIOR, D. Aptidão física relacionada à saúde de jovens cegos em escolas regulares e especiais. Revista Brasileira de Crescimento e Desenvolvimento Humano, São Paulo, v.19, n.1, p.42-53, 2009.

GUEDES, D.P.; GUEDES, J.E.R.P. Manual prático para avaliação em educação física. Barueri: Manole, 2006.

HALLAL, P.C. Padróes de atividade física em adolescentes de 10-12 anos de idade: determinantes precoces e contemporâneos. Tese (Doutorado) - Universidade Federal de Pelotas, Pelotas, 2005.

KODISH, S.; KULINNA, P.H.; MARTIN, J.; PANGRAZI, R.; DARST, P. Determinants of physical activity in an inclusive setting. Adapted Physical Activity Quarterly, Champaign, v.23, p.390-409, 2006.

KORACH, O.; TENNENBAUM, A.; SCHNITZER, R.; OMOY, A. Early motor development of blind children. Journal of Paediatrics and Child Health, Melbourne, v.36, n.3, p.226-9, 2000.

LIEBERMAN, L.J. Self-determination in physical activity: research to practice. Revista Sobama, Rio Claro, v.10, n.1, p.1-7, 2005.

LIEBERMAN, L.J.; STUART, M.E.; HAND, K.; ROBINSON, B. An investigation of the motivational effects of talking pedometers among children with visual impairments and deaf-blindness. Journal of Visual Impairment and Blindness, New York, v.100, n.12, p.726-36, 2006.

LIMA, R.B.C.P.; FERMINO, R.C.; SEABRA, A.; GARGANTA, R.; MAIA, J.A.R. Padrão de atividade física em crianças e jovens: um breve resumo do estado de conhecimento. Revista Brasileira de Cineantropometria e Desempenho Humano, Florianópolis, v.12, n.1, p.68-76, 2010.

LONGMUIR, P.E.; BAR-OR, O. Factors influencing the physical activity levels of youths with physical and sensory disabilities. Adapted Physical Activity Quarterly, Champaign, v.17, p.40-53, 2000.

LOPES, M.C.B.; KITADAI, S.P.S.; OKAI, L.A. Avaliação e tratamento fisioterapêutico das alterações motoras presentes em crianças deficientes visuais. Revista Brasileira de Oftamologia, Rio de Janeiro, v.63, n.3, p.155-61, 2004.

LOPES, V.P.; MAIA, J.A.R.; OLIVEIRA, M.M.C.; SEABRA, A.; GARGANTA, R. Caracterização da atividade física habitual em adolescentes de ambos os sexos através de acelerometria e pedometria. Revista Paulista de Educação Física, São Paulo, v.17, n.1, p.51-63, 2003.

MATSUDO, S.M.; MATSUDO, V.R.; ARAÚJO, T.; ANDRADRE, D.; ANDRADRE, E.; OLIVEIRA, L.; BRAGGION, G. Nível de atividade física da população do Estado de São Paulo: análise de acordo com o gênero, idade, nível sócioeconômico, distribuição geográfica e de conhecimento. Revista Brasileira de Ciência do Movimento, São Caetano do Sul, v.10, n.4, p.41-50, 2010.

NOCE, F.; SIMIN, M.A.; MELLO, M.T. A percepção de qualidade de vida de pessoas portadoras de deficiência física pode ser influenciada pela prática de atividade física? Revista Brasileira de Medicina do Esporte, São Paulo, v.15, n.3, p.174-8, 2009. NUNES, M.M.A.; FIGUEIROA, J.N.; ALVES, J.G.B. Excesso de peso, atividade física e hábitos alimentares entre adolescentes de diferentes classes econômicas em Campina Grande (PB). Revista da Associação Médica Brasileira, São Paulo, v.53, n.2, p.130-4, 2007.

PEIXOTO, M.R.G.; BENÍCIO, M.H.A.; JARDIM, P.C.B.V. The relationship between body mass index and lifestyle in a Brazilian adult population: a cross-sectional survey. Caderno de Saúde Pública, Rio de Janeiro, v.23, n.11, p.2696-740, 2007. 
PRESIDENT'S COUNCIL ON PHYSICAL FITNESS AND SPORTS (PCPFS). The president's challenge: physical activity and fitness awards program. Bloomington: Departament of Health and Human Services, 2004.

SKAGGS, S.; HOOPER, C. Individuals with visual impairments: a review of psychomotor behavior. Adapted Physical Activity Quarterly, Champaign, v.13, p.16-26, 1996.

STUART, M.E.; LIEBERMAN, L.; HAND, K.E. Beliefs about physical activity among children who are visually impaired and their parents. Journal of Visual Impairment and Blindness, New York, v.100, n.4, p.223-34, 2006.

WENTHE, P.J.; JANZ, K.F.; LEVY, S.M. Gender similarities and differences in factors associated with adolescent moderatevigorous physical activity. Pediatric Exercise Science, Champaign, v.21, p.291-304, 2009.

\begin{tabular}{r|r} 
ENDEREÇo & \\
Márcia Greguol & \\
Centro de Educação Física e Esporte & \\
Universidade Estadual de Londrina & Recebido para publicação: 01/ 09/2011 \\
Rod. Celso Garcia Cid PR 445km 380 - Campus Universitário & 1a. Revisão: 01/ 12/2011 \\
Caixa Postal 6001 & 2a. Revisão: 08/03/2012 \\
86051-980 - Londrina - PR - BRASIL & Aceito: 22/03/2012 \\
e-mail: mgreguol@uel.br & \\
& \\
\hline
\end{tabular}

